\title{
Bluetooth Low Energy sensor networks for railway applications
}

\author{
Ángela Hernández, Antonio Valdovinos \\ Aragon Institute for Engineering Research (I3A) \\ University of Zaragoza \\ Zaragoza, Spain
}

\author{
David Perez-Diaz-de-Cerio, Jose Luis Valenzuela \\ Signal Theory and Communications Department \\ Universitat Politècnica de Catalunya \\ Castelldefels, Spain
}

\begin{abstract}
This paper analyzes the viability of Bluetooth Low Energy (BLE) as the enabling technology in railway scenarios to allow sensor data transmission from ground to train. This proposal is based on using the broadcasting capabilities introduced by the Bluetooth specifications since v4.0. The last version, v5.0, extends the range the speed and the broadcast capacity. However, due to its recent publication it is hard to find real devices with it implemented in the market. The proposed schema has been validated by means of experimental measurements using $\mathbf{v} 4.2$.
\end{abstract}

Keywords- BLE; vehicular networks; energy consumption; railway communications

\section{INTRODUCTION}

In the last few years, Internet of the Things (IoT) has experimented a technological leap in sensor applications. The new wireless standards allow to transmit more information with less energy consumption and with more reliability. Hundreds of sensors and information systems can be integrated in the railway to continuously monitor their parameters creating intelligent transport systems. The use of wired and wireless elements for sensing and railway communications in railway lines has many challenges. Energy consumption, reliability and delay are the main parameters which limit the use of autonomous sensors.

The European Rail Traffic Management System (ERTMS) [1] or Communications-Based Train Control (CBTC) [2] have been implemented to improve the interoperability of systems and to create a standard for train control and command systems. Nevertheless, these systems lack of flexibility to install the sensors in determined conditions. The main objective of this work is to investigate the feasibility of using Bluetooth Low Energy (BLE) wireless technologies for train communication scenarios and their future integration in the railway communications systems. Many different sensors (Fig. 1) could make use of BLE as its enabling technology: railway worker protection systems, collision avoidance, meteorological sensors, track monitoring systems, train detection mechanisms, pantograph and catenary sensors, etc.

Generally, Bluetooth is ruled out as a suitable technology for vehicular communications. There is a much extended thought which says that Bluetooth is limited to a very short range and requires a considerably amount of time to stablish a connection and transmit data. In [3], it was already demonstrated that

This work has been supported in part by the MINECO/FEDER under the projects TEC2014-58341-C4-2-R and TEC2014-60258-C2-2-R. classic Bluetooth could be employed for railway communication systems. However, the internal operation and the roles of the employed devices is completely different when using BLE. In [3], the classic Bluetooth sensors required to be constantly scanning for the arrival of the convoy, which was generating remote name requests. When the sensor received this request it answered with the stored data. This mechanism, although viable, consumes a huge amount of energy on the sensor side. With the introduction of BLE, we propose to switch the roles and the place the scanner inside the train engine. Thus, the power consumption of the sensors will be reduced drastically.

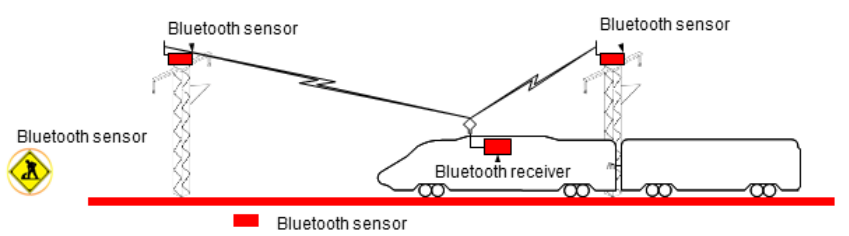

Fig. 1. Example of a railway scenario sensor deployment

So, in this paper, we will demonstrate that BLE is appropriate for this kind of communications improving the performance obtained by classic Bluetooth in terms of data reception latency and energy consumption with a similar range.

\section{BLE OVERVIEW}

The BLE specification defines a complete wireless architecture intended to enable low-power communications. BLE defines 40 broadcast and connection channels in the Industrial Scientific Medical (ISM) $2.4 \mathrm{GHz}$ band with a physical data rate of 1 Mbps.

Unlike classical Bluetooth, BLE defines three special channels (37, 38 and 39), called advertising channels used for the process of discovering, initiating a connection and broadcasting data. In this work, we used non-connectable undirected advertising, which is one of the different advertising events proposed by the standard. Thus, the advertiser periodically generates events, which consist of a sequence of packets in the three advertising channels. The period between these events is determined by the advertising interval parameter ( $T_{\text {advInterval }}$ ) plus a random period between 0 and $10 \mathrm{~ms}$ ( $\left.\tau_{\text {randomDelay }}\right)$ to avoid consecutive collisions among different advertisers. 
The total packet size is 47 bytes, where the payload is variable from eight to 39 raw bytes. However, in our experiments, the payload consists of a two-byte header plus the ADV address, a three-byte flags field to allow the reading of the advertising by standard mobile applications and the user data itself ranging from 1 to 26 bytes plus two bytes for length and type of data.

\section{EXPERIMENTAL PERFORMANCE OF BLE}

To evaluate the suitability of BLE for ground to train communications we analyzed its range, the data reception delay, its performance on high density sensor networks and the energy consumption.

\section{A. Range}

It is widely believed that Bluetooth has a limited range of tens of meters. Even the Bluetooth SIG has recently published a white paper to demystify this fact [4]. The real effective range is conditioned by the transmitted power, distance, propagation conditions, the antennae and the receiver sensitivity. Nevertheless, a quick evaluation could be done using the free space propagation model [5] or with simple measures [3]. In equation $1, \mathrm{P}_{\mathrm{R}}$ is the received power in Watts, $\lambda$ is the electromagnetic wavelength $(0.125 \mathrm{~m}), \mathrm{G}$ is the antenna gain, $d$ is distance between transmitter and receiver. The subscripts $\mathrm{T}$ and $\mathrm{R}$ denote each one of them, respectively. Using the maximum equivalent isotropically radiated power (EIRP) in the 2.4 GHz band, $100 \mathrm{~mW}$, combined both with high sensitivity receivers $(-93 \mathrm{dBm})$ and $3 \mathrm{dBi}$ receiver antenna, Bluetooth can theoretically allow ranges up to near five kilometers:

$$
P_{R}=P_{T} \cdot G_{T} \cdot G_{R} \cdot\left(\frac{\lambda}{4 \pi d}\right)^{2}
$$

However, in practice, we have measured that due to other impairments, e.g., the presence of obstacles or the effect of the ground, weather conditions, the losses introduced by both the antenna cable and the connectors, etc., the range is limited to about two kilometers.

\section{B. Data reception delay and high density sensor networks}

Another fact that should be refuted is that Bluetooth introduces too much delay on the connection and data transmission which makes it unviable for vehicular communications where high speed moving nodes are involved.

With this objective in mind, we designed a sensor network with up to 15 devices (from two to 14 advertisers and one scanner) and we measured the mean time between consecutive advertisements coming from the same device. We made these measurements for three user payloads (1, 10 and 26 bytes) and three different advertising intervals (100, 300 and $500 \mathrm{~ms})$. The results are depicted in Fig. 2. There, we can appreciate that for a couple of devices, the data can be obtained in the expected value of $\mathrm{T}_{\text {advInterval }}+\tau_{\text {randomDelay. }}$

We can extract more conclusions from this figure. For example, when the number of devices increases, the collisions and, therefore, the non-detections also increase. Nevertheless, they keep on a sustainable rate. The packet size also has a small impact on the results, the larger the packet, the more probable are the collisions. And, thus, the data reception gets slightly delayed.

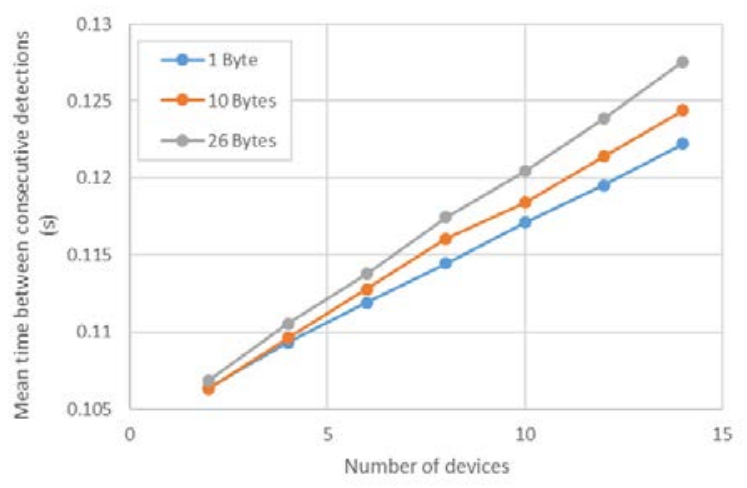

(a) $\mathrm{T}_{\text {advInterval }}=100 \mathrm{~ms}$

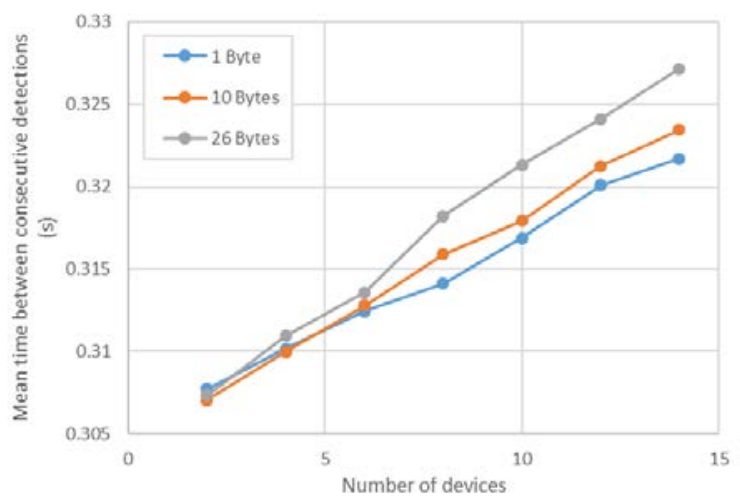

(b) $\mathrm{T}_{\text {advInterval }}=300 \mathrm{~ms}$

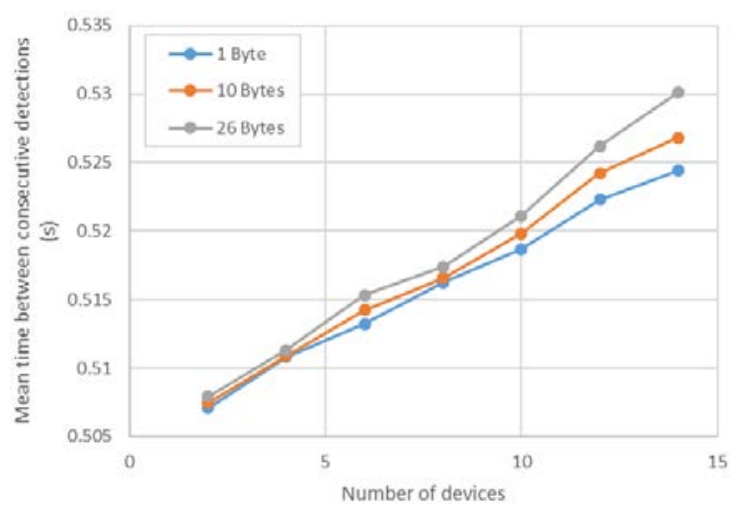

(c) $\mathrm{T}_{\text {advInterval }}=500 \mathrm{~ms}$

Fig. 2. Mean time between consecutive detections

So, the delay until we receive a packet depends not only on

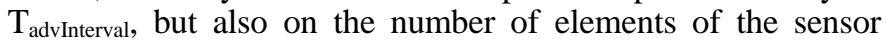
network. Fig. 3 depicts the probability density function of receiving an advertisement in a worst case scenario with 25 real advertisers using a $\mathrm{T}_{\text {advinterval }}$ of $100 \mathrm{~ms}$ and transmitting 26-byte user data packets. There, we can see that if the scanner could remain under the coverage area for at least $600 \mathrm{~ms}$ it will receive data from all the advertisers of the sensor network. 


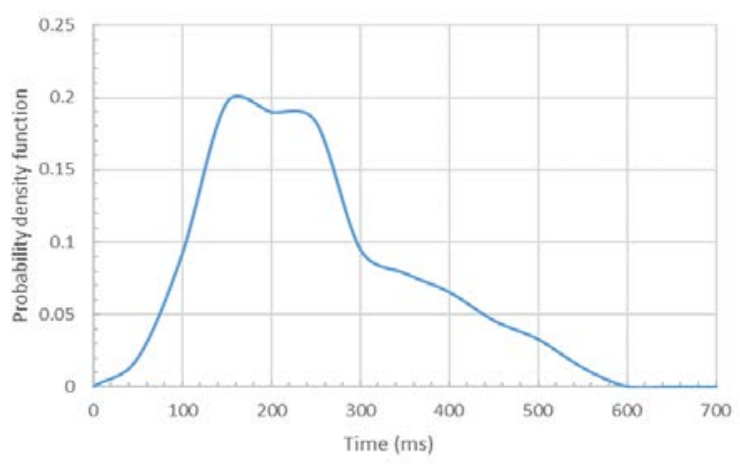

Fig. 3. Probability density function of receiving an advertisement (25 nodes)

To complement these measurements, we designed an experiment where the BLE scanner was placed in a car at 120 $\mathrm{km} / \mathrm{h}$ and then it came into the proximities of a small sensor network composed of five devices. The advertisers used a $\mathrm{T}_{\text {advinterval }}$ of 100ms. Fig. 4 depicts the results obtained. Each point in the figure represents the received signal strength indicator (RSSI) of each received advertisement. As can be seen, at this speed, the mobile node takes near ten seconds to arrive to the point nearest the sensors since it receives the first advertisement. Thus, combining these results with the previously obtained in Fig. 3, the mobile has enough time to receive the data from all the sensors and even it would be possible to reduce the advertising interval to save energy.

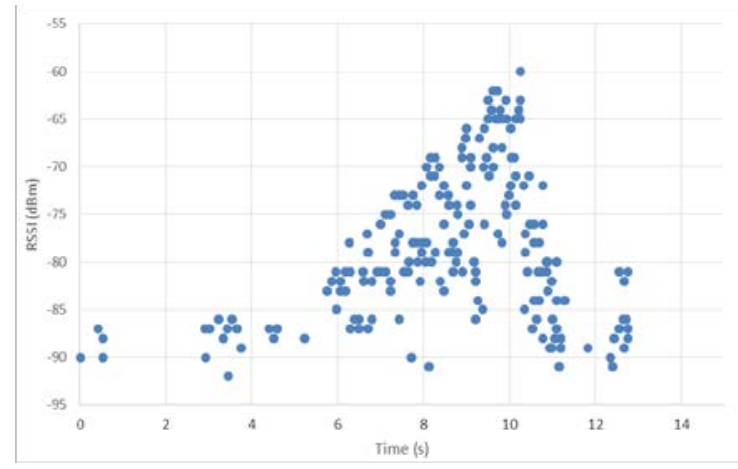

Fig. 4. RSSI of the received packets with a high speed mobile node

\section{Energy consumption}

Finally, one key aspect to discuss when talking about BLE is the energy consumption. Classic Bluetooth energy consumption is in the $\mathrm{mA}$ order of magnitude, for example, the SPBT3.0DP2 chipset from STMicroelectronics has a current consumption in inquiry and page scan mode of $9 \mathrm{~mA}$ [6].

On the other hand, for the proposed system with BLE, the average current consumption for the different employed parameters is shown in Table I. It can be noticed that the wireless sensor devices need up to 500 times less energy using BLE.

\section{CONCLUSIONS}

In this work we conducted and discussed several real experiments to demonstrate that Bluetooth is suitable for ground to train connectivity in railway communications. The presented work validates that Bluetooth Low Energy can be used as a wireless long range system, and it is possible to transmit data to high speed moving receivers.

We assumed that a 26-byte user data packet can carry enough information for railway sensor applications and, thus, the advertising procedure is proposed against other connection mechanisms. Using this procedure, a message of up to 26 bytes can be transmitted in average around 100 ms with a very low energy consumption. On the other hand, it has been demonstrated that this data exchange can be completed within wide coverage areas, up to two kilometers, with standard and commercial devices equipped with omnidirectional antennas.

Although the presented results were performed in near optimal conditions such as line of sight, we have verified that Bluetooth systems also operate properly transmitting with a leaky feeder or in bad weather conditions. Moreover, Bluetooth technology can use mesh network protocols that can extend data transmission over tens of kilometers. The results presented in this paper are obtained using real deployments. So, we can conclude that Bluetooth is a viable wireless technology for designing sensors used in complex railway communications.

TABLE I. AVERAGE CURRENT CONSUMPTION

\begin{tabular}{|c|c|c|c|}
\hline $\begin{array}{c}\text { Advertising } \\
\text { interval (ms) }\end{array}$ & $\begin{array}{c}\text { User data } \\
\text { payload } \\
\text { (bytes) }\end{array}$ & $\begin{array}{c}\text { Redbear } \\
\text { Nano }(\boldsymbol{\mu A})\end{array}$ & $\begin{array}{c}\text { Cypress } \\
\text { PSoC4-A BLE } \\
(\boldsymbol{\mu A} \mathbf{)}\end{array}$ \\
\hline 100 & 1 & 17.3 & 235 \\
\hline 100 & 10 & 18.5 & 268 \\
\hline 100 & 26 & 20 & 327 \\
\hline 300 & 1 & 10.4 & 79.24 \\
\hline 300 & 10 & 10.8 & 90.32 \\
\hline 300 & 26 & 11.5 & 110 \\
\hline 500 & 1 & 9.1 & 48 \\
\hline 500 & 10 & 9.2 & 54 \\
\hline 500 & 26 & 9.5 & 66 \\
\hline
\end{tabular}

\section{REFERENCES}

[1] S. K. Abed, "European Rail Traffic Management System - An overview," 2010 1st Int. Conf. Energy, Power Control, 2010.

[2] IEEE standards, "IEEE Standard for Communications-Based Train Control (CBTC) Performance and Functional Requirements.” .

[3] J. Higuera, E. Kartsakli, J. L. Valenzuela, L. Alonso, A. Laya, R. Martinez, and A. Aguilar, "Experimental Study of Bluetooth, ZigBee and IEEE 802.15.4 Technologies on Board High-Speed Trains,” in 2012 IEEE 75th Vehicular Technology Conference (VTC Spring), 2012, pp. 1-5.

[4] C. Coupe, S. Hegenderfer, and S. Schmidt, "Debunking the myth: Bluetooth ${ }^{\circledR}$ Range.” p. 8, 2016.

[5] H. T. Friis, "A Note on a Simple Transmission Formula," Proc. IRE, vol. 34, no. 5, pp. 254-256, May 1946.

[6] STMicroelectronics, "SPBT3.0DP2 Bluetooth ${ }^{\circledR}$ Classic module Datasheet.” 2017. 